\title{
Fourier transform ion cyclotron resonance mass spectrometry in the speciation of high molecular weight sulfur heterocycles in vacuum gas oils of different boiling ranges
}

\author{
Saroj K. Panda • Wolfgang Schrader • Jan T. Andersson
}

Received: 29 April 2008 / Revised: 16 July 2008 / Accepted: 21 July 2008 / Published online: 10 August 2008

(C) The Author(s) 2008

\begin{abstract}
The analysis of sulfur aromatics in vacuum gas oils (VGO) distilled from an Iranian light crude oil is discussed. The VGOs were fractionated into three boiling ranges, $390-460,460-520$, and $520-550{ }^{\circ} \mathrm{C}$, and were analyzed using liquid chromatographic separation on a $\mathrm{Pd}$ (II)-bonded stationary phase followed by identification with electrospray ionization Fourier transform ion cyclotron resonance mass spectrometry (FT-ICR MS). It was possible to detect a large number of thiophenes, including a substantial number of isomers, in the three VGO fractions. Separation on the palladium phase and inclusion of sulfurselective derivatization makes electrospray ionization of these nonpolar compounds possible. An elemental composition can be assigned to a large number of $S_{1}$ compounds without ambiguity in the presence of abundant hydrocarbons. With an increase in boiling temperature, an increase in the size of the aromatic system and the number of side chain carbon atoms was observed. In addition, the masses of higher magnitude shifted toward larger aromatic systems
\end{abstract}

This work is dedicated to Prof. B. Krebs on the occasion of his 70th birthday.

S. K. Panda $\cdot$ J. T. Andersson $(\bowtie)$

Institute of Inorganic and Analytical Chemistry,

University of Münster,

Corrensstrasse 30,

48149 Münster, Germany

e-mail: anderss@uni-muenster.de

S. K. Panda $\cdot$ J. T. Andersson

NRW Graduate School of Chemistry, University of Münster,

Corrensstrasse 36,

48149 Münster, Germany

W. Schrader

Max-Planck-Institut für Kohlenforschung,

45470 Mülheim an der Ruhr, Germany with an increase in boiling range. A comparison of FT-ICR MS and comprehensive gas chromatography is also given.

Keywords Fourier transform ion cyclotron resonance mass spectrometry · Crude oil analysis · Liquid chromatography . Electrospray $\cdot$ Sulfur

\section{Introduction}

High oil prices and diminishing supplies warrant a smarter use of the remaining oil resources, including more extensive use of fractions that have not been valued as highly in the past. Such cuts need more treatment in the refineries, but owing to the increased prices for crude oil lately this is now becoming economically feasible. Major efforts are directed toward reducing the content of certain heteroelements, such as sulfur, nitrogen, and metals, indicating the need for analytical methods that give reliable information on these elements in the high-boiling fractions.

The most widely used method for removal of sulfur is catalytic hydrodesulfurization, which operates at high hydrogen pressure and at elevated temperatures. Most nonthiophenic sulfur compounds react faster than thiophenic sulfur compounds [1] but there are large differences in reaction rate also among the different thiophenes, depending on the molecular structure. Lighter distillates contain predominantly the one- and two-ring thiophenes, but in the higher-boiling vacuum gas oils (VGO), larger aromatic systems with more and longer alkyl chains are present that make the hydrodesulfurization of VGOs more difficult [2]. Much research work is devoted to understanding hydrodesulfurization on a molecular level; therefore, the sulfur aromatic compounds in VGOs are an important object of study that can give essential information toward 
establishing the relationship between their molecular structure and the ease of hydrodesulfurization of VGOs.

Owing to the low volatility of high molecular weight compounds, it is difficult to analyze such polycyclic aromatic sulfur heterocycles (PASHs) in VGOs and to benefit from the high resolution offered by capillary gas chromatography (GC). Despite this, some attempts have been made and, with mass-selective detection, the molecular ions that correspond to several series of PASHs were found. In a VGO (b.p. $300-500{ }^{\circ} \mathrm{C}$, sulfur content $1.7 \mathrm{wt} \%$ ), alkylated derivatives of benzothiophene and dibenzothiophene as well as of the benzonaphthothiophenes were detected. The temperature limitation of approximately $300{ }^{\circ} \mathrm{C}$ for $\mathrm{GC}$ restricted the study to compounds with molecular masses up to approximately $300 \mathrm{Da}$ [3]. In addition to the same series of compounds, also alkylated phenanthro[4,5-bcd]thiophenes were reported in a VGO of boiling range from 340 to more than $530{ }^{\circ} \mathrm{C}$ [4]. They were analyzed by GC-mass spectrometry (MS) after separation from the polycyclic aromatic hydrocarbons (PAHs) on a $\mathrm{PdCl}_{2}$ phase. Again, the choice of $\mathrm{GC}$ as the separation technique limited the usefulness of the method to compounds having a mass of less than $304 \mathrm{Da}$, which corresponds to $\mathrm{C}_{5}$-benzonaphthothiophenes, but compounds of masses well over $300 \mathrm{Da}$ are expected to be numerous in VGOs (see later).

Liquid chromatography does not suffer from this limitation due to volatility but it does not show the high resolution offered by GC. Coupled with mass-spectrometric techniques, it can offer a valuable avenue for exploring compounds with a molecular mass above $300 \mathrm{Da}$. In previous studies we separated the aromatic components of crude oil fractions on a stationary phase containing $\mathrm{Pd}(\mathrm{II})$ complexed by 2 -amino1-cyclopent-1-enedithiocarboxylate that was covalently bonded to a silica gel. This phase provides more favorable properties [5] than the initially used $\mathrm{PdCl}_{2}$ phase [6, 7]. PAHs show insignificant interactions with $\mathrm{Pd}$ (II) and are generally eluted in a first fraction. In lighter petroleum cuts such as gas oils and middle distillates, there is a clean separation of PAHs and PASHs [5], but a vacuum residue showed a different behavior [8] in that a range of PASHs were eluted in the first fraction. Experiments with a large number of synthesized reference compounds of the PASH class [9] have shown that thiophenes attached to one or more aromatic rings in a noncondensed way are found in the first fraction, whereas the second fraction contains thiophenes with fused aromatic rings, e.g., benzothiophenes and higher homologues.

There is no universally applicable analytical technique to analyze all components of a crude oil, but methods are available that allow such materials to be analyzed in quite some depth although, depending on the method used, they rarely reveal the whole picture. Fourier transform (FT) ion cyclotron resonance (ICR) MS has an unsurpassed ability to characterize thousands of components in a complex petroleum mixture by combining high resolution and mass accuracy $[10,11]$. FT-ICR MS with electrospray ionization (ESI) was used to extract information on a large number of polar components $[10,12,13]$ which are not amenable to analysis with GC owing to their low volatility. The characterization of nonpolar compounds containing sulfur, relatively more abundant in high-boiling fractions, is not achievable with ESI FT-ICR MS. Therefore, a technique was implemented to selectively derivatize such heterocycles into thiophenium salts using methyl iodide [8]. This procedure allows the use of ESI for the analysis of PASHs. Recently, continuous-flow field desorption [14] and lowvoltage electron ionization [15] coupled with FT-ICR MS were reported for the study of VGOs of different origin and of different boiling ranges, respectively. Other reports [16] compared the use of the derivatization method using ESI detection with atmospheric pressure photoionization (APPI) and indicated that the range in the first case mentioned was limited to double bond equivalent (DBE) values below 15 and therefore the APPI approach was favored. It has to be noted here that the chemical derivatization process generates salts which are in fact solution-phase ions that are useful for ESI without further solvent modifiers. The use of a thermal nebulizer in APPI sets limits for the suitability of this technique and therefore APPI does not give a complete picture of the components of a crude oil, it just shows another part of it. The combination of two-dimensional liquid chromatographic separation and derivatization, as used here, allows the selective characterization of sulfur-containing compounds. Nitrogen heterocycles and hydrocarbons are not present in the liquid chromatography fractions methylated and thus the technique is highly selective toward sulfur aromatic compounds.

In this work we attempt a characterization of the PASHs in a VGO, distilled from an Iranian light crude oil, that was fractionated into three boiling ranges: $390-460,460-520$ and $520-550{ }^{\circ} \mathrm{C}$. Of particular interest was to clarify if there is a change in the chemical composition of the samples with boiling range, i.e., if other ring systems prevail, or if the major difference would lie in the number of substituent carbon atoms of the aromatic systems. A characterization of the VGO fractions was thus carried out using multiple steps of chromatographic sample preparation followed by FT-ICR MS analysis, yielding information that can be used for optimization of catalytic hydrodesulfurization processes.

\section{Experimental}

Three Iranian light VGO samples of different boiling ranges (390-460, 460-520, and $520-550{ }^{\circ} \mathrm{C}$ ) were supplied by the 
Institut Français du Pétrole, Vernaison, France. The VGOs had a sulfur content of $1.12,1.33$, and $1.77 \mathrm{wt} \%$, respectively. Chemicals for synthesis and high performance liquid chromatography grade solvents were from SigmaAldrich (Taufkirchen, Germany). Separation of the aliphatic and aromatic fractions was achieved on an open tubular column packed with a mixture of $20 \mathrm{~g}$ silica gel and $35 \mathrm{~g}$ alumina (both activated at $180{ }^{\circ} \mathrm{C}$ for $16 \mathrm{~h}$ ). The aliphatics were collected using $120 \mathrm{~mL} n$-heptane as the eluent and the aromatics were eluted by $360 \mathrm{~mL}$ of an $n$-heptane-toluene mixture $(2: 1 \mathrm{v} / \mathrm{v})$. The detailed analysis scheme is presented in Fig. 1 .

Ligand exchange chromatography

The Pd(II)-bonded silica gel was synthesized with $10 \mu \mathrm{m}$ LiChrosorb Si 100 (Merck, Darmstadt, Germany) as described in the literature [17-19]. The bonded silica was packed in a stainless steel column $(250 \mathrm{~mm} \times 8 \mathrm{~mm})$ at a pressure of 350 bar using a Knauer pneumatic pump. The first fraction (Pd-1) was eluted with cyclohexane-dichloromethane $(7: 3 \mathrm{v} / \mathrm{v})$ and the second fraction (Pd-2) was eluted after addition of $0.5 \%$ 2-propanol to the previous mobile phase. The flow rate was maintained at $3 \mathrm{~mL} / \mathrm{min}$ throughout the whole separation.

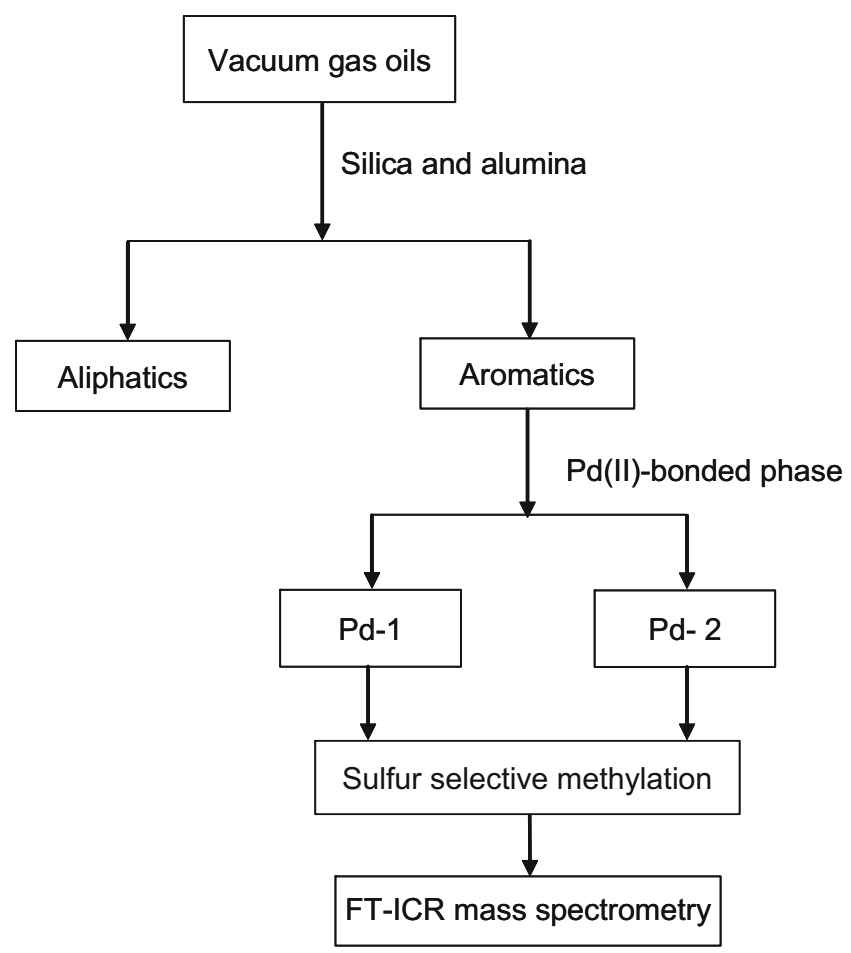

Fig. 1 Analysis scheme for sulfur aromatics present in the three vacuum gas oils. $P d-1$ first fraction, $P d-2$ second fraction, $F T-I C R$ Fourier transform ion cyclotron resonance
Gas chromatography-mass spectrometry

High-temperature GC-MS measurement was carried out using an HP 5890 II gas chromatograph with a Finnigan MAT SSQ $7000 \mathrm{MS}$ detector. The capillary column was a VF-5 HT $(10 \mathrm{~m} \times 0.25 \mathrm{~mm} \times 0.25 \mu \mathrm{m})$. The injector temperature was set to $300{ }^{\circ} \mathrm{C}$ and electron ionization was performed at $70 \mathrm{eV}$. The temperature program was $50{ }^{\circ} \mathrm{C}$ at $0 \mathrm{~min}, 8.6^{\circ} \mathrm{C} / \mathrm{min}$ to $180^{\circ} \mathrm{C}, 7.1^{\circ} \mathrm{C} / \mathrm{min}$ to $230^{\circ} \mathrm{C}$, and $8.5^{\circ} \mathrm{C} /$ $\min$ to $400{ }^{\circ} \mathrm{C}$.

Sulfur-selective methylation

About $50 \mathrm{mg}$ of the aromatic fraction from each VGO was separated on a $\mathrm{Pd}(\mathrm{II})$-bonded stationary phase into two fractions: Pd-1 and Pd-2. To each fraction an excess of methyl iodide and silver tetrafluoroborate in 1,2-dichloroethane was added. The mixture was allowed to react for $48 \mathrm{~h}$, following which the precipitate was removed by filtration and washed with 1,2-dichloroethane. Finally, the methyl thiophenium salts were obtained through evaporation of the filtrate [8].

\section{High-resolution MS}

Mass spectra were acquired using an APEX III FT-ICR mass spectrometer (Bruker Daltonics, Bremen, Germany) equipped with a 7-T actively shielded superconducting magnet and an Agilent ESI source. The thiophenium ions were dissolved in a 1:1 (v/v) solution of dichloromethaneacetonitrile and injected in the infusion mode with a flow rate of $2 \mu \mathrm{L} / \mathrm{min}$. The spray voltage was maintained at $4.5 \mathrm{kV}$. The ions were accumulated for $0.5 \mathrm{~s}$ in the octapole before transfer to the cyclotron cell. For a better signal-tonoise ratio, at least 64 scans were accumulated. Internal and external calibrations were performed using Agilent electrospray calibration solution with masses 322.04812, 622.02896, and 922.00980 .

Assignment of compound classes from accurate mass data

All the data obtained from the mass spectrometer were imported into an Excel spreadsheet. To assign the elemental composition of the original components, all the masses obtained as $\left[\mathrm{M}+\mathrm{CH}_{3}\right]^{+}$were converted to neutral masses by subtracting 15.02293 from the measured masses. Then IUPAC masses were converted to the Kendrick mass scale by the following equation [20]:

Kendrick mass $=$ IUPAC mass $\times(14.00000 / 14.01565)$.

In this study, the masses were separated into different homologues by their nominal masses and Kendrick mass 
defect following a multiple sorting procedure [21]. The relationship between the Kendrick mass defect and the Kendrick nominal mass is as follows:

Kendrick mass defect $=$ Kendrick nominal mass

$$
\text { - Kendrick exact mass. }
$$

For simplicity, the DBE concept was used instead of Kendrick mass defects in all figures containing Kendrick plots to convey essential information about the number of rings and double bonds without going into detail about Kendrick mass defects. The DBE always represents the sum of the total number of rings and double bonds present in a compound [11].

\section{Results and discussion}

The standard method for the analysis of crude oil components is GC, preferably with a mass-selective detector to increase the amount of information that can be gathered. Even high-temperature GC allows no more than a partial separation of the complex mixture of VGOs, so the gain in information is modest, as can be seen in Fig. 2. This figure shows a small fraction of the whole VGO, namely, only those PASHs that are found in Pd-2. Despite this, the number of compounds is so huge that a fairly featureless hump is observed in the total ion trace. Extracting the signals for $m / z 316$ gives a chromatogram with some resolved peaks sitting on top of an unresolved hump. This mass corresponds to the molar mass of $\mathrm{C}_{13}$-benzothiophenes which are expected to be numerous in the low-boiling VGO. A mass spectrum recorded at the retention time for the apex of the dominating peak shows a large number of ions. These data indicate that even after gas chromatographic separation the information in one single spectrum is overwhelming owing to several factors: a range of compounds of different molar masses as well as a number of structurally different compounds of the same mass are coeluted.

Thus, there is abundant evidence of a large number of aromatic parent systems as well as isomers of their alkylated derivatives that can be expected in VGOs and other high boiling petroleum cuts and they are eluted at exactly the same time. Despite its high resolving power, one-dimensional GC is not able to resolve these compounds sufficiently for a more detailed analysis to be carried out. Two-dimensional GC $(\mathrm{GC} \times \mathrm{GC})$ has found wide use for petroleum samples in the last few years, but to be effective the second phase must be polar to complement the first, nonpolar phase. Polar phases generally are much more thermally sensitive than nonpolar ones and would hardly stand up to the temperatures needed for samples such as high-boiling VGOs. (For a further comparison of $\mathrm{GC} \times \mathrm{GC}$ and FT-ICR MS, see " $\mathrm{GC} \times \mathrm{GC}$ and FT-ICR MS - a theoretical comparison" below.)

This is where a combination of liquid chromatography and FT-ICR MS can help in the characterization of samples such
Fig. 2 Gas chromatographymass spectrometry chromatogram of Pd-2 of the low-boilingrange vacuum gas oil (blue). Extracted ion chromatogram of $\mathrm{m} / \mathrm{z} 316$ corresponding to $\mathrm{C}_{13^{-}}$ benzothiophenes (red). Mass range 100-380 of the spectrum measured at $11.21 \mathrm{~min}$ (black) showing multiple components present. TIC total ion chromatogram

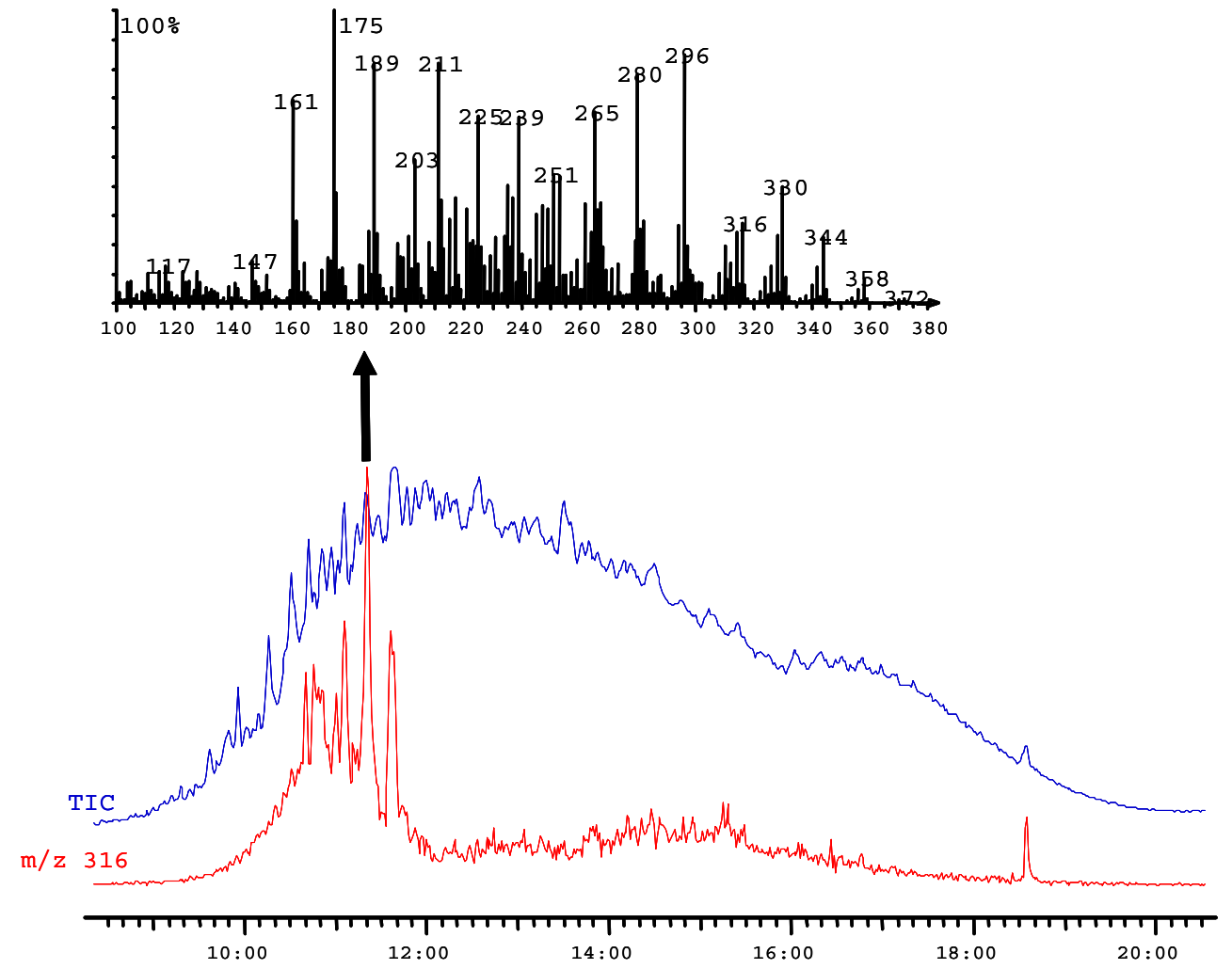


Table 1 Assigned $S_{1}$ compounds in both fractions of the three vacuum gas oils

\begin{tabular}{lll}
\hline & Pd-1 & Pd-2 \\
\hline Low boiling range & 11 & 188 \\
Medium boiling range & 94 & 225 \\
High boiling range & 106 & 288 \\
\hline
\end{tabular}

$P d-1$ first fraction, $P d-2$ second fraction

as VGOs. Following the separation on the Pd(II) column, the PASHs in the two fractions collected were methylated and investigated by FT-ICR MS. The number of PASHs in Pd-1 was less than that in Pd-2 for each boiling range, as is illustrated in Table 1 and Figs. 3 and 4, but it increased with increasing boiling temperature. The complexity of the sulfurenriched Pd-2 remains very high even after some PASHs have been separated into Pd-1 (Fig. 4). The number of assigned $\mathrm{S}_{1}$ compounds (compounds containing one sulfur atom) from Pd-1 was 11, 94, and 106 in the three boiling ranges in increasing order of distillation temperature, and the number of $\mathrm{S}_{1}$ compounds in Pd-2 was 188, 225, and 281, respectively. This corroborates the trend of an increasing number of $\mathrm{S}_{1}$ members with increasing boiling temperature as was observed by low-voltage electron ionization FT-ICR MS [15].

Information on the molecular weight distribution pattern is given by the number-average molecular weight, $M_{\mathrm{n}}$, and the weight-average molecular weight, $M_{\mathrm{w}}$. These numbers were calculated for $\mathrm{S}_{1}$ compounds in $\mathrm{Pd}-2$ of all three VGOs [22]. The mass distribution pattern for the lowboiling range $\left(M_{\mathrm{n}} / M_{\mathrm{w}}=358 / 366\right)$ was centered at about $360 \mathrm{Da}$, for the middle boiling range (423/434) at about $430 \mathrm{Da}$, and for the higher boiling range (508/519) at about $510 \mathrm{Da}$. These numbers show how the centered mass shifts toward higher masses with an increase in boiling range. These values are higher than those reported for the whole aromatic fraction of VGOs of another origin and other distillation ranges [14]. The highest-boiling VGO in that study had a similar boiling range as the two highest-boiling VGOs here but had a number-average molecular weight of 336 (vs. 423 and 508 here). This comparison shows that VGOs from different sources can have quite different distributions of substituted aromatics, thus making it difficult to use conclusions derived from one set of VGOs for the study of another set.
Fig. 3 Broad-band mass spectra of Pd-1 from three vacuum gas oils of different boiling ranges

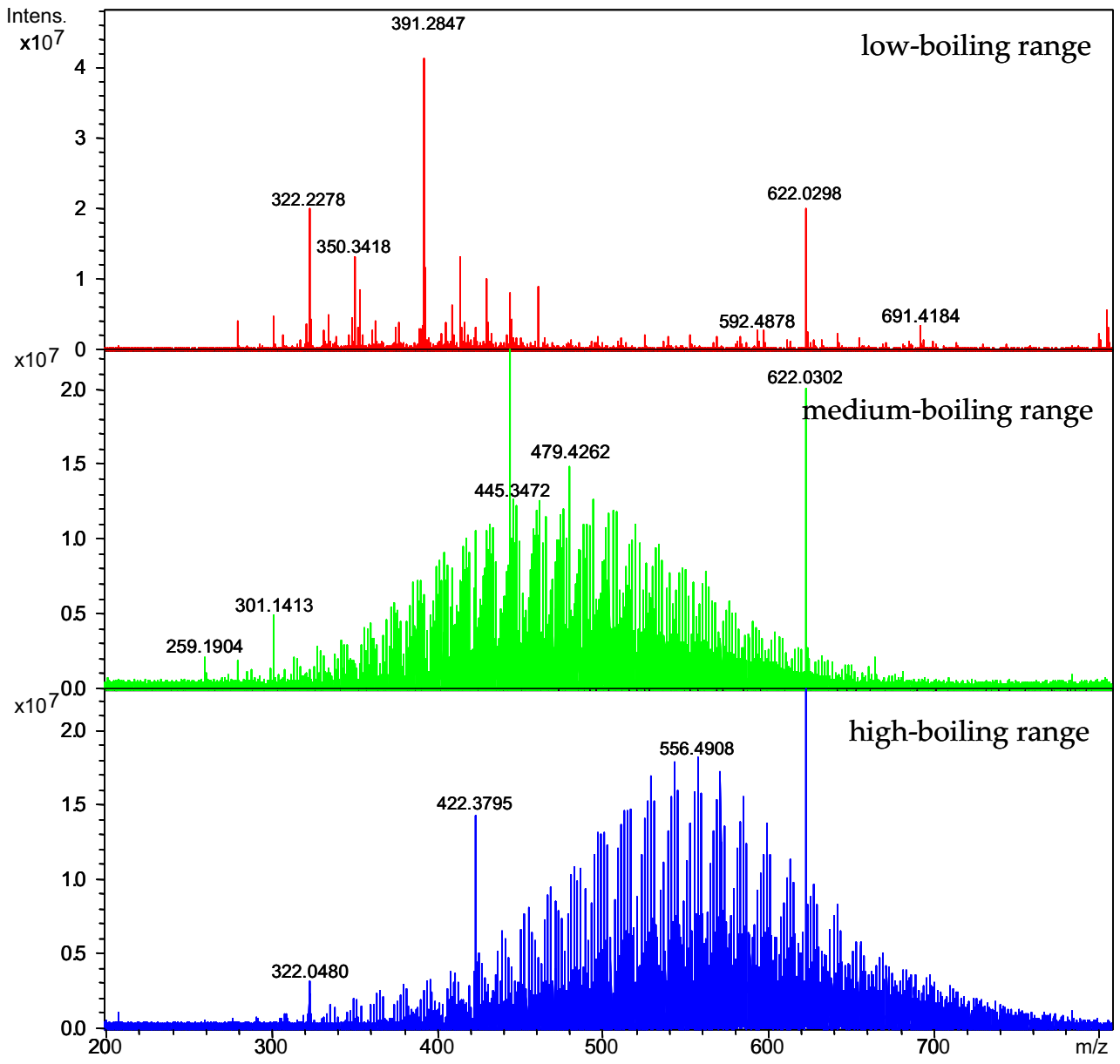


Fig. 4 Broad-band mass spectra of Pd-2 from three vacuum gas oils of different boiling ranges

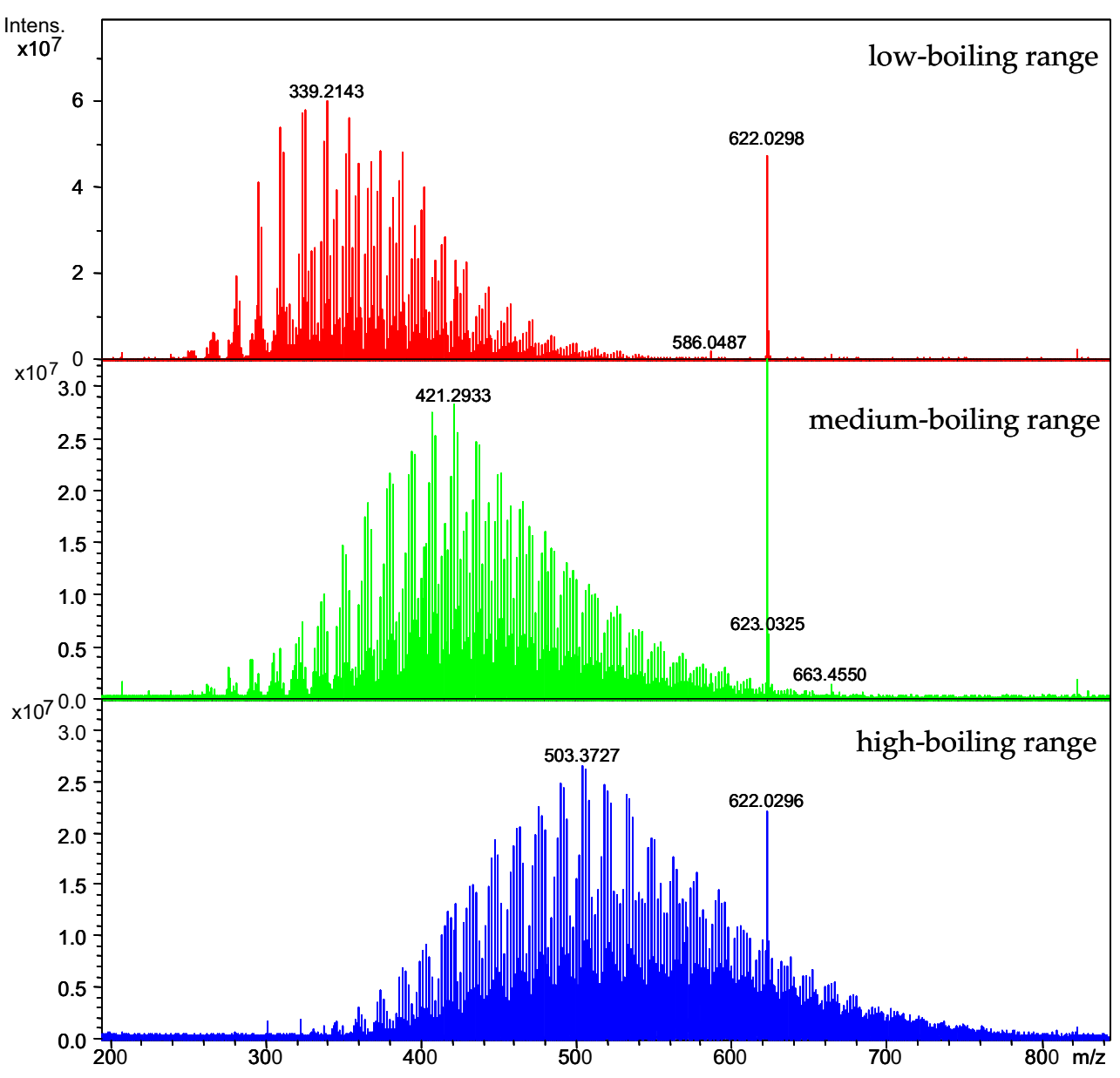

Some structural information can be deduced from the $\mathrm{DBE}$ and from the retention on the $\mathrm{Pd}(\mathrm{II})$ phase and will be discussed next.

\section{Low-boiling-range VGO}

The number of $\mathrm{S}_{1}$ compounds in Pd-1 was negligible and their relative abundance was low (Fig. 3). DBEs 3, 4, and 5, eluted in Pd-1, could correspond to thiophenes, naphthenothiophenes, and cycloalkadienothiophenes. Retention studies of model thiophenic compounds on the Pd(II) phase show that thiophenes that are not fused with another aromatic ring are not retained on this phase [9]. However, some compounds present in Pd-1 showed DBEs 6 and 7 with a mass range from 350 to $450 \mathrm{Da}$. The compounds of DBE 6 and 7 are suggested to be perhydrophenanthrothiophenes and phenylthiophenes, among other possibilities (Table 2).

The number of $\mathrm{S}_{1}$ compounds in $\mathrm{Pd}-2$ was considerably higher than in Pd-1 (Table 1). Here, the masses range from 220 to $550 \mathrm{Da}$, with the highest DBE observed being 13 . Additionally, some compounds of DBEs 3, 4, and 5 of minor intensities were detected, as shown in Fig. 5, possibly indicating the presence of alkylated thiophenes, alkylated naphthenothiophenes, and alkylated cycloalkathiophenes. The signals at DBE 6 range from 288 to $540 \mathrm{Da}$ and represent major constituents. It is reasonable to postulate that they correspond to benzothiophenes with 11-29 $\mathrm{CH}_{2}$ units. The intense signals in the mass range 300-400 Da indicate a high abundance of those alkylated compounds in the sample, although the relative abundance of ions in mass spectra does not necessarily correlate exactly with the relative abundance of the compounds in the original mixture. DBE 7 could correspond to tetrahydrodibenzothiophenes with six to 25 alkyl carbons. The abundant masses fall in the same mass range as that of the benzothiophene series.

The next-higher row of homologues shows a DBE of 8 and could correspond to indenothiophenes and indanylthiophenes or compounds from the DBE 6 and 7 rows that possess two and one more naphthenic rings, respectively. The relative abundance of the representatives of this class is lower, as indicated in Fig. 5. DBE 9 most probably represents alkylated dibenzothiophenes (or naphthothiophenes) with three to 25 alkyl carbon atoms. DBE 10 could represent acenaphthenothiophenes or dibenzothiophene with one naphtheno ring. Phenylbenzothiophenes also display this DBE value. As the DBE increases, the 
Table 2 Examples of sulfur-containing compounds that would be eluted in Pd-1 on the Pd(II) phase

$\begin{aligned} & \text { Double } \\ & \text { bond } \\ & \text { equivalent }\end{aligned}$
Compound type

number of possible parent structures increases even more, making it difficult to identify the basic ring structure.

\section{Medium-boiling-range VGO}

There are many more $\mathrm{S}_{1}$ compounds in $\mathrm{Pd}-1$ compared with the low-boiling VGO fraction. The range of DBEs goes from 3 to 9 with masses from about 250 to $550 \mathrm{Da}$. The signals of the DBE 5 and 6 series show the largest magnitude. Parent structures in this fraction can be suggested on the basis of the liquid chromatographic retention on the $\mathrm{Pd}(\mathrm{II})$-bonded stationary phase as shown in Table 2. In Pd-2 it was possible to observe a larger number of members in addition to a significant change in
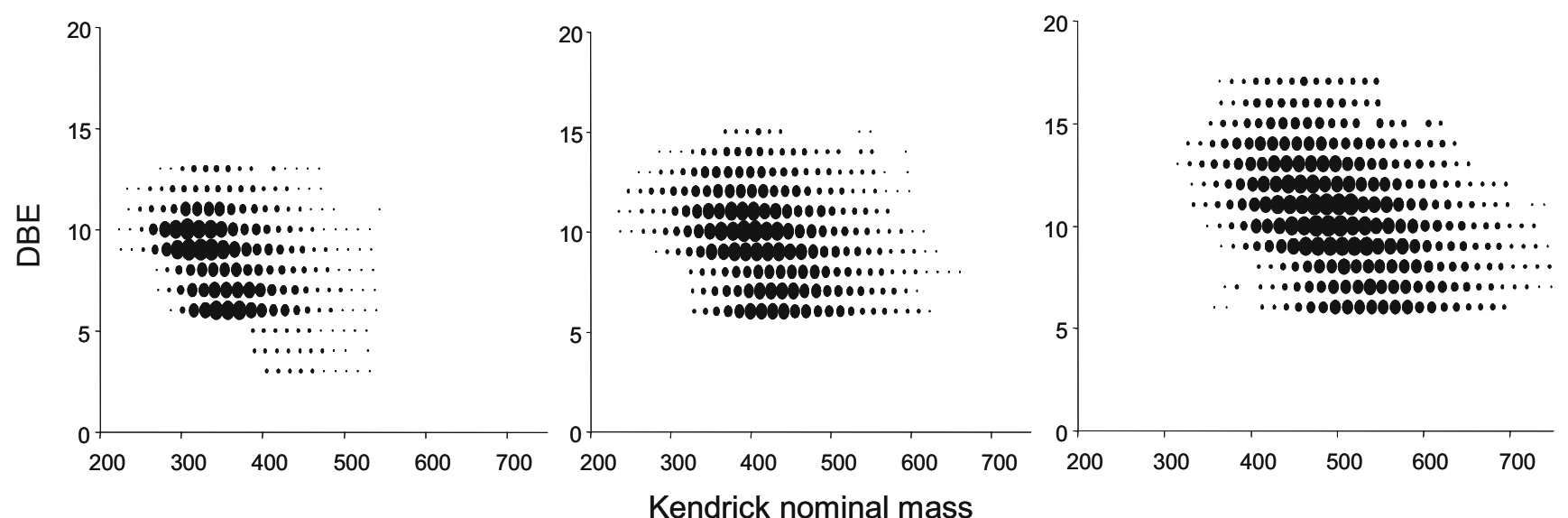

Fig. 5 Kendrick plots of Pd-2 for the low-boiling range (left), the medium-boiling range (middle) and the high-boiling range (right) of the vacuum gas oil. $D B E$ double bond equivalent 
the distribution pattern along different DBEs compared with that of the low-boiling VGO (Fig. 5). Not only is the highest observed DBE 15, compared with 13 for the lowboiling $\mathrm{VGO}$, but the relative abundance pattern across the DBE rows has been shifted toward higher numbers. The dominating series are formed by DBEs 9,10 , and 11 . With an increase in boiling range, the complexity of the aromatic systems increases with respect to both higher mass (about $650 \mathrm{Da}$ ) and the variety of aromatic parent structures (DBE attains values from 6 to 15). Here, the initial members for DBEs 6,7 , and 8 are found at about $330 \mathrm{Da}$, whereas the ones from the low-boiling range are at about $270 \mathrm{Da}$. In this fraction DBE 13 is more abundant compared with the previous VGO, with masses going up to $596 \mathrm{Da}$. The two highest DBEs, i.e., 14 and 15, were not detected in the lowboiling VGO.

\section{High-boiling-range VGO}

In Pd-1, the number of assigned $\mathrm{S}_{1}$ compounds was not significantly larger than in the medium-boiling VGO but the distribution pattern along the DBE rows was remarkably different. The compounds extend from DBE 6 to 13 with masses from 280 to about $720 \mathrm{Da}$, with the most abundant series being DBE 9 to 11 . The trend of shifting masses toward higher DBEs with increase in boiling temperature is observed here too. In Pd-2, the relative abundance of compounds shifts to a great extent toward higher DBEs. Figure 5 shows that the most intense series are formed by the PASHs of DBEs 9 through 13, followed by DBEs 6,7 , and 8 . The highest DBE in this boiling range is 17 , with a mass range of about $300-750 \mathrm{Da}$. At higher boiling ranges, therefore, the small increase in boiling temperature is reflected in a significant change toward higher masses and, less markedly, higher DBE.

In general, DBEs 6, 9, and 12, can be assigned to benzothiophenes, dibenzothiophenes, and benzonaphthothiophenes, respectively. A pseudogram of the different boiling ranges is displayed in Fig. 6, which shows the DBE 12 homologues. The pseudogram illustrates the homologue series of a Kendrick plot in more detail and was generated using nominal masses and the relative intensity of the class of compounds of interest [23]. The first signal of the lowboiling fraction corresponds to $\mathrm{m} / \mathrm{z} 234$, showing the unsubstituted benzonaphthothiophene(s).

Structural information cannot necessarily be inferred from mass-spectrometric data alone; therefore, the combined analysis of a VGO using chromatography, UV spectroscopy, and FT-ICR MS can be adopted to assign aromatic ring sizes up to DBE 12 [24]. Additionally, variations in the distribution pattern and size of the alkyl portion of these aromatic compounds can be monitored as a function of boiling range owing to their presence in all three VGOs. Furthermore, the pseudograms (Fig. 6) show that for a certain mass, e.g., 402, that corresponds to $\mathrm{C}_{12^{-}}$ benzonaphthothiophenes, representatives are found in all three distillation fractions. Therefore, isomers of widely different boiling points must occur in the VGOs, in line with the broad elution range in GC for a defined mass (Fig. 2). The reactivity toward hydrodesulfurization decreases from benzothiophene to benzonaphthothiophene to dibenzothiophene [25], but it also varies depending on the substitution pattern; therefore, detailed information on these three major classes is needed for tuning the hydrotreatment process.

The combination of chromatographic separation with mass-spectrometric detection has the additional advantages of reducing the complexity of the sample. The accuracy of data from FT-ICR MS measurements is strongly dependent on the ion load of the cyclotron cell where ion charge effects from a large loading can reduce the resolution and consequently, owing to bad peak definition, the accuracy. This is especially difficult to maintain with very complex samples, where stronger peaks can interfere with smaller signals that are of analytical interest. While ICR instruments with a higher magnetic field strength offer higher ion loading capabilities and maximum resolution, the 7-T magnet used for these studies yields better performance with the introduction of less complex samples and the results are easier to interpret in terms of structural information.

\section{$\mathrm{GC} \times \mathrm{GC}$ and FT-ICR MS—a theoretical comparison}

Previous authors have noted a parallel between the twodimensional representation of the data from high-resolution

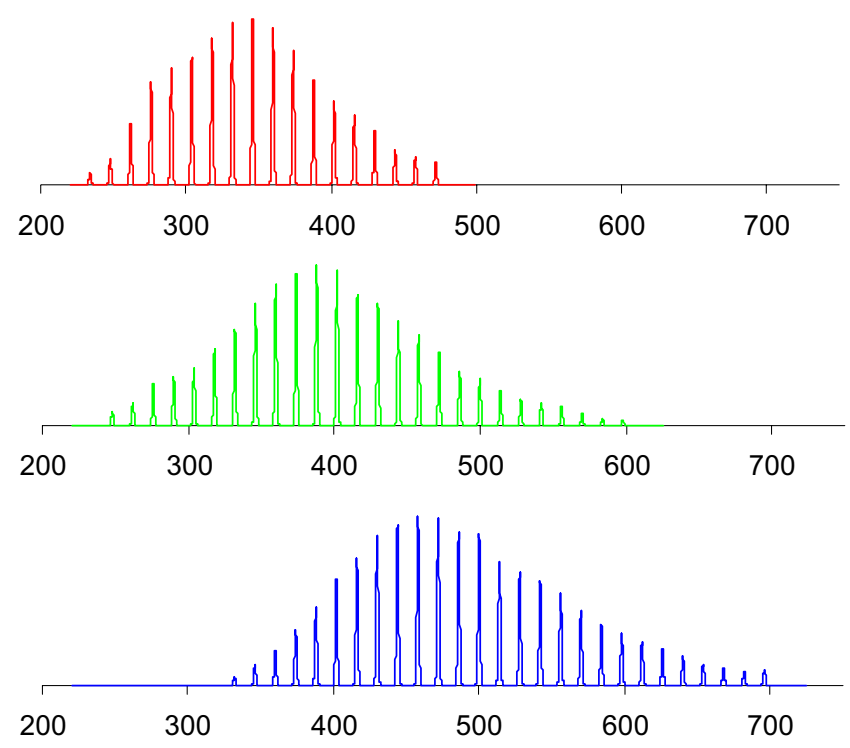

Fig. 6 Distribution pattern of alkylated benzonaphthothiophenes in the three vacuum gas oils of different boiling ranges. To $p$ low-boiling range, middle medium-boiling range, bottom high-boiling range 
optical spectroscopy and the high-resolution MS possible with FT-ICR MS [26]. We would like to point out that there is a similarity to comprehensive $\mathrm{GC}$ or $\mathrm{GC} \times \mathrm{GC}$ also. In that technique, two capillary columns are used in series. The compounds eluted from the first column, in general a capillary of normal length containing a nonpolar stationary phase, are frozen out in a transfer device. After they have been collected for a few seconds, they are quickly transferred onto the second column through heating of the transfer device. The second column is usually much shorter than the first one and contains a more polar stationary phase. The chromatography on the second column must be fast and can only take as long as the eluent of the first column is collected in the transfer device. The detector after the second column records a chromatogram every couple of seconds and the chromatograms are plotted as a common two-dimensional chromatogram, where the $x$-axis corresponds to the chromatography on the first column and the $y$-axis to the chromatography on the second column.

Since the stationary phase in the first column is nonpolar, it separates the components largely according to their vapor pressure, which-for nonpolar analytes such as the ones expected in a nonpolar fraction from petroleum samples - correlates with their molecular mass. The second stationary phase is more polar and therefore shows stronger interactions with polarizable groups, for instance, aromatic rings. If such a gas chromatogram had been possible with the very heavy compounds investigated here, a twodimensional plot would have resulted in similarities to the Kendrick plots: along the $x$-axis, the components appear in order of increasing molecular mass in both the $\mathrm{GC} \times \mathrm{GC}$ plot and the FT-ICR mass spectrum plot (Fig. 3). Along the $y$ axis the components would be chromatographically sepa- rated according to their increasing polarity: open-chain aliphatics $<$ cycloalkanes $<$ dicycloaliphatics $<$ monoaromatics $<$ diaromatics, etc. This is exactly the order that is found when they are plotted according to the DBE in a Kendrick plot from FT-ICR MS data. The effect is clearly visible in Fig. 7, illustrating a diesel sample from the literature, when compared with the Kendrick plots in Fig. 5. An advantage of the MS method is of course that it can be used for compounds of negligible volatility, whereas GC is limited to compounds of sufficient volatility, and therefore molecular weight, to pass through the column. Many of the compounds investigated in this work would not be expected to be amenable to $\mathrm{GC}$ analysis.

Naturally, the two techniques also show differences. Isomers are not resolved by MS but can be resolved by GC. Each dot in a Kendrick plot represents all compounds of the same elemental composition, but many of them would be expected to be eluted at somewhat different retention times from a GC column, yielding several peaks or at least a broadened peak for the isomers.

\section{Conclusions}

The detailed analysis of VGOs of different boiling ranges based on liquid chromatography and FT-ICR MS presents an overview of the types of sulfur aromatics in each fraction. Although the sulfur content in the three VGOs differed by no more than $0.65 \mathrm{wt} \%$, the change in relative abundance of masses along different DBEs was remarkably differentiated with the increase in boiling ranges. The isomers of the $\mathrm{S}_{1}$ compounds found in $\mathrm{Pd}-1$ after a separation on a $\mathrm{Pd}(\mathrm{II})$ phase were less numerous than the
Fig. 7 A two-dimensional gas chromatogram of a diesel. (Reprinted from [27], with permission from Elsevier)

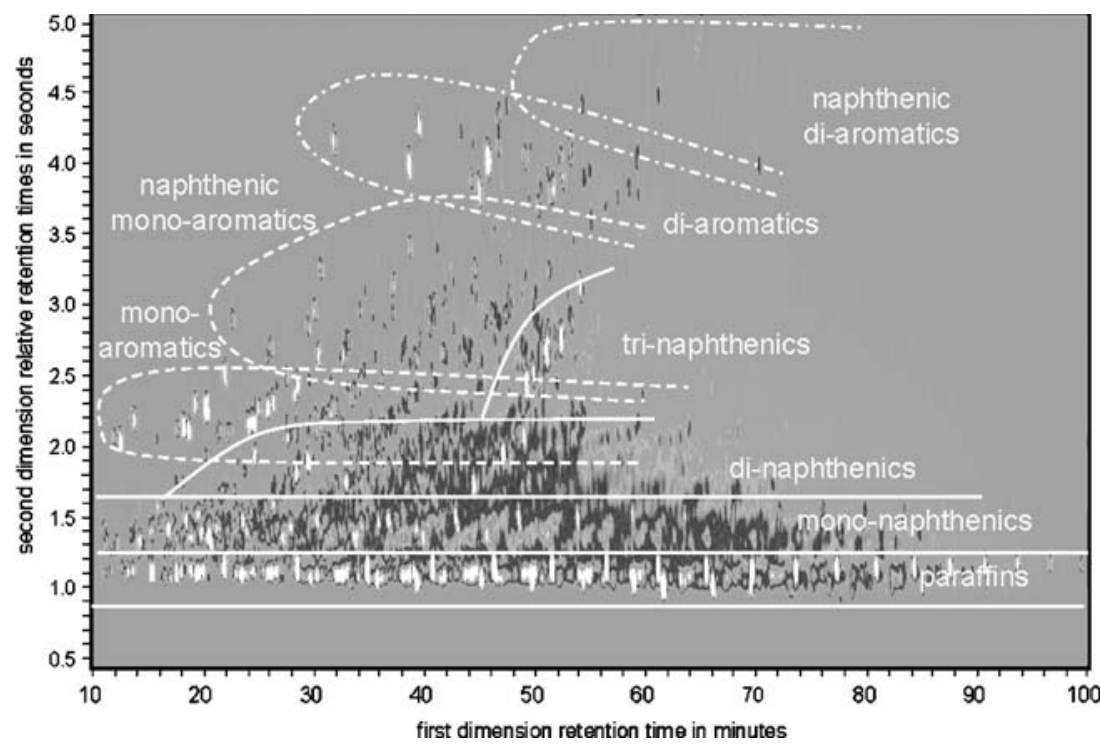


ones in Pd-2. They probably possess parent ring systems different from those of the compounds in Pd-2. This conclusion is made possible owing to their different liquid chromatographic properties on this particular phase. The combination of liquid chromatography and FT-ICR MS can therefore be a valuable tool for the speciation of sulfur compounds found in catalytically cracked VGOs. This, in turn, may help to evaluate the efficiency of catalysts used for the hydrotreatment processes.

Acknowledgements We would like to thank A. Ranc, Institut Français du Pétrol (IFP), Vernaison, France, for providing the VGOs, W. Joppek and M. Scheppat are thanked for their support with GC-MS measurement, and J. Bloomberg is thanked for permission to use Fig. 7.

Open Access This article is distributed under the terms of the Creative Commons Attribution Noncommercial License which permits any noncommercial use, distribution, and reproduction in any medium, provided the original author(s) and source are credited.

\section{References}

1. Schulz H, Ousmanov F, Waller P (1999) Fuel Process Technol 61:5-41

2. Ma XL, Sakanishi K, Isoda T, Mochida I (1995) Ind Eng Chem Res 34:748-754

3. Sugimoto Y, Tunnukij K, Miki Y, Yamadaya S (1992) Sekiyu Gakkaishi 35:339-348

4. Ma XL, Sakanishi K, Isoda T, Mochida I (1997) Fuel 76:329-339

5. Schade T, Roberz B, Andersson JT (2002) Polycyclic Aromat Compd 22:311-320

6. Andersson JT (1987) Anal Chem 59:2207-2209
7. Nishioka M, Campbell RM, Lee ML, Castle RN (1986) Fuel $65: 270-273$

8. Mueller H, Andersson JT, Schrader W (2005) Anal Chem 77:2536-2543

9. Sripada K, Andersson JT (2005) Anal Bioanal Chem 382:735-741

10. Hughey CA, Rodgers RP, Marshall AG (2002) Anal Chem 74:4145-4149

11. Panda SK, Andersson JT, Schrader W (2007) Anal Bioanal Chem 389:1329-1339

12. Qian K, Rodgers RP, Hendrickson CL, Emmett MR, Marshall AG (2001) Energy Fuels 15:492-498

13. Qian KN, Robbins WK, Hughey CA, Cooper HJ, Rodgers RP, Marshall AG (2001) Energy Fuels 15:1505-1511

14. Schaub TM, Rodgers RP, Marshall AG (2005) Energy Fuels 19:1566-1573

15. Fu JM, Kim S, Rodgers RP, Hendrickson CL, Marshall AG, Qian KN (2006) Energy Fuels 20:661-667

16. Purcell JM, Juyal P, Kim DG, Rodgers RP, Hendrickson CL, Marshall AG (2007) Energy Fuels 21:2869-2874

17. Pyell U, Stork G (1992) Fresenius J Anal Chem 342:281-286

18. Seshadri T, Kettrup A (1982) Fresenius Z Anal Chem 310:1-5

19. Takeshim $T$, Yokoyama M, Imamoto $T$, Akano $M$, Asaba H (1969) J Org Chem 34:730-732

20. Kendrick E (1963) Anal Chem 35:2146-2154

21. Hsu CS, Qian K, Chen YC (1992) Anal Chim Acta 264:79-89

22. Schaub TM, Rodgers RP, Marshall AG, Qian K, Green LA, Olmstead WN (2005) Energy Fuels 19:1566-1573

23. Panda SK, Schrader W, al-Hajji A, Andersson JT (2007) Energy Fuels 21:1071-1077

24. Panda SK, Schrader W, Andersson JT (2006) J Chromatogr A 1122:88-96

25. Choudhary TV, Malandra J, Green J, Parrott S, Johnson B (2006) Angew Chem Int Ed 45:3299-3303

26. Hughey CA, Hendrickson CL, Rodgers RP, Marshall AG, Qian KN (2001) Anal Chem 73:4676-4681

27. Edam R, Blomberg J, Hanssen H-G, Schoenmakers PJ (2005) J Chromatogr A 1086:12-20 